\title{
Early Detection of Psychosis: Challenges and Opportunities
}

\author{
Raquel E. Gur
}

Published online: 3 April 2014

(C) Springer International Publishing AG 2014

\begin{abstract}
Early identification of individuals at clinical risk for psychotic illness is critical for early intervention. Current studies apply special assessment measures combined with neurocognitive, neuroimaging, and electrophysiology methodologies that have been discovered largely in schizophrenia research. While still limited in sample size, harmonized measures, and longitudinal data, these studies indicate the presence of abnormalities in the clinical-risk state. Such findings suggest that the onset of the psychotic process precedes late adolescence and clinical detection. Efforts at early identification, therefore, could benefit from large-scale studies. Computerized clinical assessment and neurocognitive testing are available and can move from academic sites to the community. Neuroimaging methods are increasingly available and can add to the prediction of transition. As a field, we need to move vigorously and responsibly toward early interventions that may prevent and ameliorate the developmental trajectory that leads to the emergence of a full psychotic disorder with devastating impact on individuals, families and communities.
\end{abstract}

Keywords Clinical risk · Early identification .

Neurocognition $\cdot$ Neuroimaging

\section{Introduction}

The principle re-crafted by Benjamin Franklin that "an ounce of prevention is worth a pound of cure" has been the holy grail of medicine since its inception and is about to become a reality for multiple medical disorders. Progress in medicine has emphasized the importance of early identification of disorders with the goal of prevention and intervention that may reduce

\section{R. E. Gur $(\bowtie)$}

Neuropsychiatry Section, Department of Psychiatry, University of

Pennsylvania Perelman School of Medicine, 3400 Spruce Street, 10

Gates Pavilion, Philadelphia, PA 19104, USA

e-mail: raquel@upenn.edu the risk of disease onset and palliate the course and consequences of illness. Examining the disease process has shifted attention to early phases of a pathological course rather than the point at which diagnosis is made. For example, increased understanding of genetic and environmental risk factors has advanced treatment of cardiovascular disorders, where myocardial infarction is considered the culmination of multiple modifiable risk factors. Similarly, discoveries of biomarkers in cancer have advanced targeted therapeutic choices.

Psychiatry has long recognized the chronic course of schizophrenia and the enormity of its impact on affected individuals, families, and communities. However, the shift to early identification of psychosis, before the individual meets diagnostic criteria as defined by standard classifications, has been long in coming and is still controversial $[1-3,4 \bullet \bullet]$. Two complicating factors in the case of psychosis hinder the motivation for early detection both among care providers and help seekers. The first is stigma associated with psychosis. The pediatrician will have little hesitation in referring for further evaluation a child who presents with signs of diabetes or asthma but may hesitate about the implications of referring a child for evaluation of psychotic-like manifestations; children and caregivers are correspondingly reluctant to disclose such symptoms. Secondly, there are no early treatments with demonstrated effectiveness in delaying or ameliorating disease course. Therefore, the search for populations at risk needs to be population-based in addition to relying on convenience samples of help-seeking individuals. Such a goal may seem difficult to attain given the costs of screening and is questionable at this stage, given the small proportion of at-risk individuals who eventually reach diagnostic criteria for the illness. However, advances in neuroscience and technology make it increasingly feasible to screen large populations and identify those at risk at an increasingly early age. This review highlights progress in the study of early phases of psychosis including clinical manifestations and investigation of behavioral domains related to brain function. Special emphasis on the challenges and clinical implications will follow. 


\section{The Continuum of Psychosis}

Current diagnostic formulations require meeting specific criteria for schizophrenia spectrum disorders $[5,6]$. This dichotomous categorization (Fig. 1) does not fully reflect the process where the presentation of psychotic symptoms is likely the final outcome that can be assessed more reliably and upon which therapeutic interventions are based. Historically, positive symptoms of hallucinations and delusions have been the focus of characterization and treatment and only more recently have the negative symptoms associated with schizophrenia received increasing attention as an important component of the disorder.

Examination of the biological process of psychosis raises questions of how early we should start and what gradation should be applied to determine the level of risk for psychosis. Furthermore, as only a portion of individuals who present with sub-threshold psychotic symptoms transition to psychotic illness, what are the predictors of those who move in the continuum, depicted in Fig. 2, to more severe symptoms and those whose symptoms subside? Having this information will be important and help care providers, affected individuals, and families. To answer these fundamental questions requires systematic research, and a growing literature has contributed to the accumulating body of knowledge relevant to the questions raised.

\section{Clinical Risk}

The impetus for research on early identification stems from the devastating effects of schizophrenia on multiple domains including cognitive, social, and vocational functioning. Notably, considerable decline occurs early in the disease course $[7,8]$ and early identification and intervention is essential for amelioration and possible prevention of the full impact of psychosis. Criteria for psychosis risk have been defined and applied globally in multiple studies that included

\section{Diagnostic approach Liability approach}

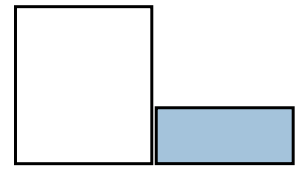

Not Affected Affected Discrete

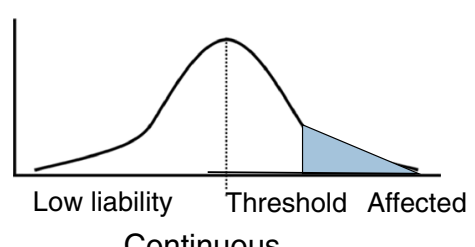

Continuous
Fig. 1 Illustration of the difference between the traditional diagnostic approach (left) and the current dimensional liability approach to psychosis (right). The diagnostic approach requires 'cases' to meet specific criteria and the analyses are done on discrete categorization of the sample into those not affected and those who are ill. The liability approach considers continuous quantitative measures that reflect the risk of developing disease

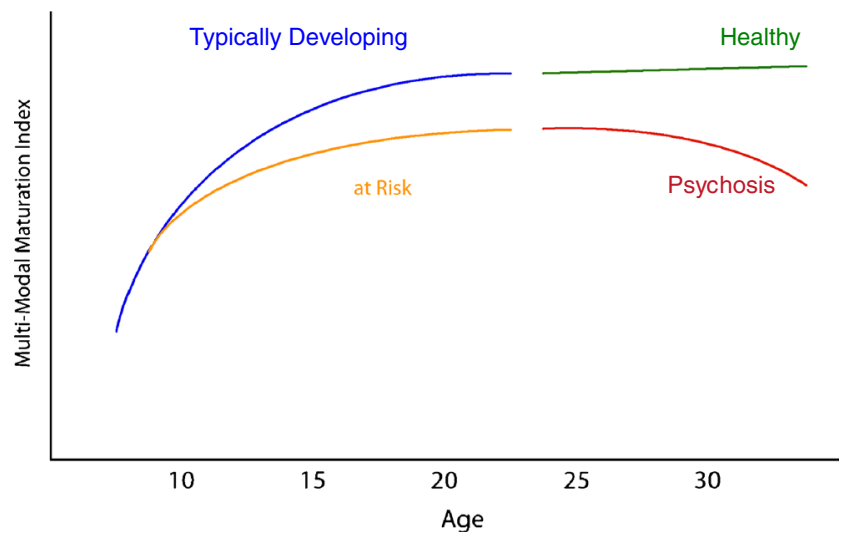

Fig. 2 Developmental trajectories: multiple indices of brain function show age-related maturation and should provide early markers of vulnerability to psychosis. (Adapted from T. Satterthwaite and R.E. Gur, University of Pennsylvania)

help-seeking individuals [9-11]. The clinical presentation is characterized by a recent onset of sub-threshold psychotic symptoms that are associated with distress and functional impairment. There is increased risk for progression along the psychosis continuum and transition to a psychotic disorder in the $2-3$ years after meeting criteria for clinical risk $[12,13]$.

The established procedures and criteria provided by the Structured Interview for Prodromal Syndromes (SIPS) and similar instruments have enabled a common approach in research on sub-threshold psychotic symptoms $[9,14,15]$. The SIPS is a well validated instrument and inter-rater reliability is excellent. The criteria for the clinical high-risk state predict about $20 \%$ conversion to psychosis at 1 year and about $35 \%$ at 3 years $[14,15]$. The SIPS provides 19 subscales comprising the Scale of Prodromal Symptoms (SOPS) that are theoretically grouped into positive, negative, disorganized, and general domains, with gradation of severity optimally centered on sub-threshold levels [14]. Within a single academic center and across collaborating centers, training in administration, probing, and scoring are critical for establishing reliability among assessors. These procedures will require special training of mental-health experts and should become part of the clinical training of mental-health professionals.

As progression to psychotic illness occurs in only a portion of individuals who meet at-risk state criteria, the potential contribution of other measures has been considered. This approach is consistent with other complex medical disorders, where multiple risk factors contribute to emergence of disease. For example, in cardiac disorders, family history, hypertension, increased cholesterol, diabetes, obesity, sedentary lifestyle, and stressors are all factors evaluated.

Considering the accumulating literature $[4 \bullet \bullet, 12]$ and the burden of psychotic disorders, the Diagnostic and Statistical Manual of Mental Disorders (DSM)-5 Psychotic Disorders Work Group reviewed the body of evidence, obtained input 
from leading authorities and the public, and, following extensive discussions, recommend the inclusion of 'attenuated psychosis syndrome' as a clinical category with specific criteria in the appendix of DSM-5 as a condition that merits further study [16•], as outlined below.

The Clinical Criteria for Attenuated Psychosis Syndrome (APS)

A. Presence of symptoms

- At least one of the symptoms below is present in attenuated form with sufficient severity and/or frequency to warrant clinical attention.

1. Delusions/delusional ideas

2. Hallucinations/perceptional abnormalities

3. Disorganized speech/communication

B. Frequency and duration of symptoms

- The symptoms must be present at least once per week for the past month.

\section{Symptoms onset and course}

- The symptoms must have begun or worsened in the past year.

\section{Symptoms impact}

- The symptoms are sufficiently distressing and disabling leading to help seeking.

\section{E. Consideration of other potential diagnosis}

- The symptoms are not better explained by any other DSM-5 diagnosis, such as substance-related disorders, and clinical criteria for a psychotic disorder have never been met [17].

The debate on whether the clinical-risk state should be included as a major diagnostic category in the DSM-5 was affected by several factors including evidence that about a third of individuals at the prodromal phase of illness convert to psychotic illness in the upcoming 2-3 years; the comorbidity of mood, anxiety, and substance use disorders that is common in young people and is likely to impact the clinical presentation; and the need for further work and training when translating the efforts from academic sites to clinical settings. Furthermore, early diagnosis with uncertainty regarding future course can have a negative impact and be associated with increased anxiety and stigma.
Advanced neuroscience methods applied to elucidate the neurobiology of schizophrenia have been extended to the study of high-risk samples to evaluate whether the patterns of brain dysfunction manifested in schizophrenia are already evident in at-risk individuals. Such studies also assessed the extent to which neurocognitive and neuroimaging measures add to predicting future clinical course, specifically transition to psychosis.

\section{Neurocognition}

A core feature of schizophrenia is impaired cognition that is associated with poorer functioning and outcome [18, 19]. As cognitive deficits are present already at the first episode of psychosis $[20,21,22 \bullet \bullet$, neurocognitive assessment with measures established in investigations of schizophrenia has been applied to individuals at risk for psychosis [22••, 23•, 24, 25]. Studies vary in ascertainment approach, clinical assessment instruments applied, existence of comorbid conditions, and neuropsychological tests used. Nonetheless, metaanalyses indicate impairment in several cognitive domains that are present in at-risk individuals. For example, one meta-analysis [24] reported small to medium effect sizes of neurocognitive impairment in the psychosis risk group, with effects in general cognitive abilities, language functions, episodic memory, attention, visuo-spatial abilities, and working memory. Notably, follow-up studies where the clinical status was re-evaluated showed that individuals in the psychosis risk group who later transitioned to psychosis had greater neurocognitive deficits at baseline compared with healthy participants, with medium to large effect sizes.

Similarly, another meta analysis also reported neurocognitive deficits in the clinical-risk group, compared with healthy controls, in several domains including general intelligence, executive functions, attention, working memory, verbal fluency, verbal and spatial memory and social cognition [25]. In a portion of the studies with longitudinal data, transition to psychosis was examined and, again, impaired baseline performance in several neurocognitive domains was associated with transition to psychosis.

While the samples are still relatively small and the period of follow-up is limited and variable with respect to participant retention and treatment, the data indicate the utility of cognitive evaluation at first presentation of individuals at clinical risk. Indeed, lower intellectual functioning is associated with risk for schizophrenia spectrum disorders and is evident before the onset of psychosis [21, 26-28]. Thus, the disabling impact of the at-risk state may be attributed to impaired cognition that affects school performance and vocation at a critical time of adolescence and young adulthood when transition to independence unfolds. 
Negative symptoms have major impact on social functioning and outcome in schizophrenia [29, 30]. The extension of research effort to clinical risk along the psychosis continuum has focused on cognitive measures. Few studies have examined the domain of social cognition and those did report significant impairment in at-risk samples [31,32]. These early studies encourage further research that can link to negative symptoms and appropriate interventions aimed at their amelioration.

The literature on clinical-risk studies is based on helpseeking individuals and indicates that neurocognitive impairment is evident at time of conferring the clinical-risk state. A complementary line of research on populationbased studies confirms the association between the presence of sub-threshold psychotic symptoms and cognitive deficits [13, 27, 33, 34•, 35-38]. Epidemiologic studies report the presence of psychotic symptoms in $17 \%$ of children, ages 9-12 years [37], and $7.5 \%$ of adolescents, ages 13-18 years. Most studies have not evaluated concomitant cognitive performance.

We established a developmental cohort with concurrent evaluation of psychotic symptoms and neurocognitive performance [39]. This cross-sectional study identified that youths who reported symptoms associated with psychosis showed neurocognitive delay already at age 8 (Fig. 3). Of note, the delay is especially pronounced for complex cognition and social cognition and minimal for episodic memory and sensorimotor speed, suggesting areas for targeted interventions that can capitalize on relatively preserved functional domains. Longitudinal studies are necessary to determine whether the combination of clinical and neurocognitive measures can help narrow the population identified as at risk and improve prediction to the point where intervention can be economically feasible. Notably, this study used a 1-hour web-based battery that can be administered in multiple settings and is publicly available.
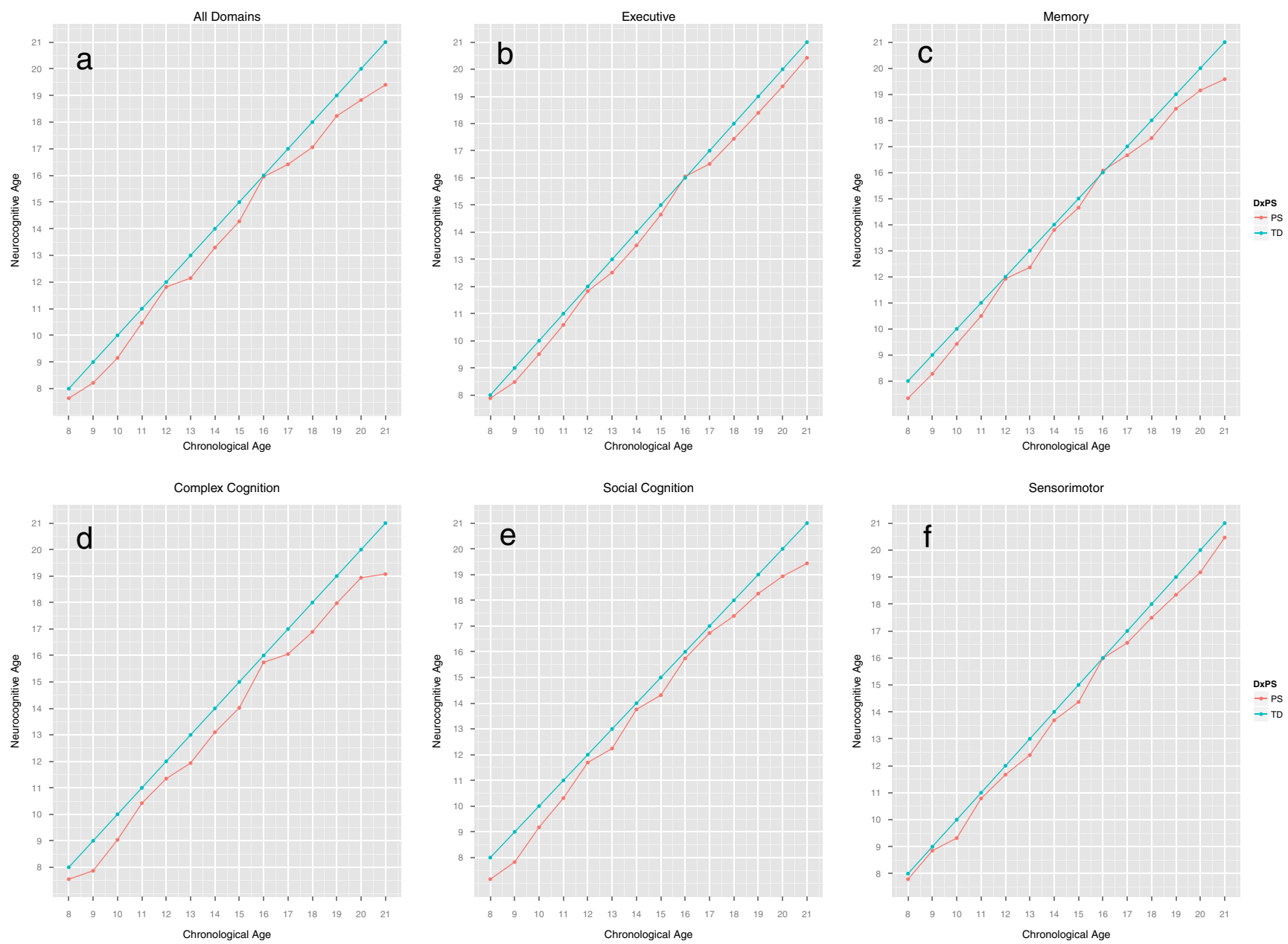

Fig. 3 Growth chart of neurocognitive development. Chronological age is compared with predicted neurocognitive age in years for typically developing ( $T D$; blue line), and psychosis spectrum ( $P S$; red line) youths.

Growth charts are provided for (a) Predicted age based on all scores (all domains), (b-f) predicted age based on tests grouped by each of the five domains assessed 


\section{Neuroimaging}

\section{Structural and Functional MRI}

Multi-modal examination of brain structure and function has provided important details on brain nodes and systems implicated in schizophrenia. Diffuse abnormalities are already present in first-episode psychosis [40], so extending such studies along the psychosis continuum to include individuals at clinical risk can provide important information on brain aberrations associated with the emerging psychotic process.

The structural MRI literature on clinical risk for psychosis [41] includes relatively small and heterogeneous samples and limited longitudinal studies. A meta-analysis of voxel-based morphometry studies compared psychosis risk $(n=198)$ with healthy controls $(n=254)$ and first-episode patients with schizophrenia $(n=206)$ with healthy controls $(n=202)$ [40]. Gray matter volume was lower in several regions including the right temporal, limbic, and prefrontal cortex in the clinicalrisk group, and in the temporal insular cortex and cerebellum in the first-episode group. Psychosis onset was associated with decreased gray matter volume in temporal, anterior cingulate, cerebellar, and insular regions. These regions have been implicated in schizophrenia and their integrity is required for cognition and emotion processing. The results suggest that brain regions that show volume reduction in schizophrenia also show abnormalities in those at risk for psychosis [41]. Effect sizes are modest, reflecting the heterogeneity of the limited samples and measures applied. Larger samples in a longitudinal design will be important to advance the understanding of underlying neuroanatomical differences between groups. White matter changes have also been reported in schizophrenia, early in the course of illness, as well as in individuals at risk for psychosis [42, 43].

Even more limited is the literature on diffusion tensor imaging (DTI) in psychosis risk. Several cross-sectional studies reported reduced fractional anisotropy in the frontal lobe [44] and in the superior longitudinal fasciculus [45]. A longitudinal study [43] compared individuals at risk for psychosis $(n=32)$, healthy controls $(n=32)$, and first-episode patients with schizophrenia $(n=15)$. The psychosis risk group was intermediate, with decreased fractional anisotropy and increased diffusivity.

Functional MRI (fMRI) has been applied to individuals at risk for psychosis, commonly in small samples with tasks that have shown differences between patients with schizophrenia and healthy participants. For example, applying a working memory paradigm showed that individuals at risk for psychosis had decreased activation in the blood oxygenation leveldependent (BOLD) response in dorsolateral and medial prefrontal regions [40]. The aberrant activity was less pronounced than the patterns observed in schizophrenia. We found a similar effect in our study of youths with psychosis

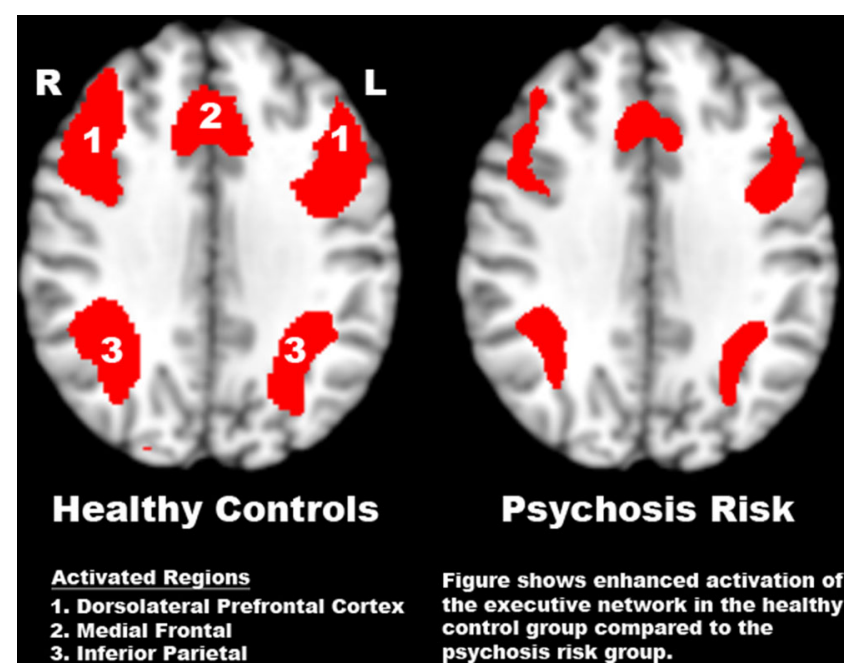

Fig. 4 Functional MRI (fMRI) blood oxygenation level-dependent (BOLD) activation in response to a working memory task. A normal pattern of activation (left) and reduced activity in clinical risk for psychosis (right) illustrates the pattern observed in studies applying the n-back paradigm. DLPFC dorsolateral prefrontal cortex

sub-threshold symptoms (Fig. 4). Few longitudinal studies examined the effects of disease progression on fMRI abnormalities [46]. This small literature suggests that individuals who transition to psychosis differ from those who do not, with the latter group showing normalization. Thus, the application of fMRI holds promise as a tool that may facilitate elucidation of the underlying pathophysiology of the psychotic process.

The resting BOLD signal in fMRI paradigms can provide a measure of neural network connectivity, reflecting 'cross-talk' integration among brain regions. It examines the time-series correlations among brain regions, indicating which regions show synchronized activation. Aberrations in schizophrenia in fronto-temporal connectivity have been reported and have also been seen in those at clinical risk [47]. At present, this literature is too limited to derive any reasonable conclusions.

\section{Neurotransmitters}

Several neurotransmitters that have been related to the pathophysiology of schizophrenia have been examined in people at risk for psychosis. Dopamine dysregulation has been linked to psychosis, and positron emission tomography (PET) studies have shown increased dopamine striatal activity in schizophrenia [48-50]. Striatal 6-fluoro-L-dopa F18-dopa was also elevated in psychosis-risk individuals and related to symptom severity [51].

Glutamate has also been implicated in the pathophysiology of schizophrenia and studies have examined individuals at genetic risk for psychosis using magnetic resonance 
spectroscopy (MRS). Increased glutamine/glutamate ratio in medial frontal cortex was reported in adolescents at genetic risk [51]. In a study integrating fMRI and MRS, 24 psychosisrisk individuals were compared with 17 healthy controls [52]. BOLD response to a verbal fluency task showed that the psychosis risk group had greater bilateral activation than controls in the mid-frontal gyrus. Glutamate levels in the thalamus were lower in psychosis-risk individuals. Furthermore, the pattern of correlations with activation suggests that prefrontal, hippocampal, and temporal functioning are related to thalamic glutamate levels and differentiate those at risk from controls.

\section{Electrophysiology}

Initial efforts applying electrophysiological paradigms to the study of clinical risk examined P300 amplitude alone or in combination with structural MRI. Thus, a study of a clinicalrisk sample [53] reported reduced amplitude in the lefthemisphere temporo-parietal region as well as in sagittal midline electrodes. In an integrative P300 and MRI, gray matter volume reported concomitant amplitude and volume decrease in multiple, primarily frontal, brain regions. A small sample was followed longitudinally and the emergence of psychosis was associated with further decrease in gray matter volume but no further changes in P300 amplitude [54].

Event-related potential (ERP) paradigms have been well established in schizophrenia research. Mismatch negativity (MMN) amplitude is reduced in schizophrenia as well as in clinical-risk samples [55, 56], suggesting that aberrations are evident before the onset of psychosis. Furthermore, transition to psychosis was associated with greater deficits in baseline measures of MMN in the clinical risk group [56, 57]. These early efforts are promising and more data are needed.

\section{Conclusions}

Early identification is essential because of the devastating effects of schizophrenia on multiple domains including cognitive, social, and vocational functioning. The considerable decline that occurs early in the disease course makes early identification and intervention vital for amelioration and possible prevention of the full impact of psychosis.

Based on the available literature, family history and clinical features associated with psychosis and impaired social functioning are important as predictors of transition to a psychotic disorder. Neurocognitive measures add potential predictors but their value needs to be further established in large-scale longitudinal studies. Neuroimaging and electrophysiology measures are potential second-tier tools that require additional research. Integration across these domains in sufficiently powered genotyped samples followed prospectively will give clinical neuroscience the necessary rungs to climb the ladder leading to improved early identification and intervention.

\section{Compliance with Ethics Guidelines}

Conflict of Interest Raquel Gur has no potential conflicts of interest relevant to this article to report.

Human and Animal Rights and Informed Consent This article does not contain any studies with human or animal subjects performed by any of the authors.

\section{References}

Papers of particular interest, published recently, have been highlighted as:

- Of importance

-• Of major importance

1. Van Os J, Linscott R, Myin-Germeys I, et al. A systematic review and meta analysis of the psychosis continuum: evidence for a psychosis proneness-persistence-impairment model of psychotic disorder. Psychol Med. 2009;39:179-95.

2. Carpenter WT, van Os J. Should attenuated psychosis syndrome be a DSM-5 diagnosis? Am J Psychiatry. 2011;168:460-3.

3. McGorry P, van Os J. Redeeming diagnosis in psychiatry: timing versus specificity. Lancet. 2013;381:343-5.

4.• Fusar-Poli P, Carpenter WT, Woods SW, McGlashan TH. Attenuated psychosis syndrome: ready for DSM-5.1? Annu Rev Clin Psychol. 2014. A comprehensive and thoughtful review of the current state of knowledge and issues related to the inclusion of the attentuated psychosis syndrome in the next update of DSM-5.

5. Diagnostic and statistical manual of mental disorders: Washington, DC: American Psychiatric Association 2013. Psychiatr Publ 5th Ed.

6. Heckers S, Barch DM, Bustillo J, et al. Structure of the psychotic disorders classification in DSM-5. Schizophr Res. 2013;150:11-4.

7. Isohanni M, Murray GK, Jokelainen J, et al. The persistence of developmental markers in childhood and adolescence and risk for schizophrenic psychoses in adult life. A 34-year followup of the Northern Finland 1966 birth cohort. Schizophr Res. 2004;71:213-25.

8. Woodberry KA, Seidman LJ, Giuliano AJ, et al. Neuropsychological profiles in individuals at clinical high risk for psychosis: relationship to psychosis and intelligence. Schizophr Res. 2010;123:188-98.

9. Miller TJ, McGlashan TH, Rosen JL, et al. Prodromal assessment with the Structured Interview for Prodromal Syndromes and the Scale of Prodromal Symptoms: predictive validity, inter rater reliability, and training to reliability. Schizophr Bull. 2003;29:703-15.

10. Yung AR, Yuen HP, McGorry PD, et al. Mapping the onset of psychosis: the comprehensive assessment of at-risk mental states. Aust N Z J Psychiatry. 2005;39:964-71.

11. Riecher-Rossler A, Aston J, Ventura J, et al. The Basel Screening Instrument for Psychosis (BSIP): development, structure, reliability and validity. Fortschr Neurol Psychiatr. 2008;76:207-16. 
12. Fusar-Poli P, Borgwardt S, Bechdolf A, et al. The psychosis highrisk state: a comprehensive state-of-the-art review. JAMA Psychiatry. 2013;70:107-20.

13. Ruhrmann S, Schultze-Lutter F, Salokangas RK, et al. Prediction of psychosis in adolescents and young adults at high risk: results from the prospective European prediction of psychosis study. Arch Gen Psychiatry. 2010;67:241-51.

14. Miller TJ, McGlashan TH, Rosen JL, et al. Prospective diagnosis of the initial prodrome for schizophrenia based on the Structured Interview for Prodromal Syndromes: preliminary evidence of interrater reliability and predictive validity. Am J Psychiatry. 2002;159:863-5.

15. Woods SW, Addington J, Cadenhead KS, et al. Validity of the prodromal risk syndrome for first psychosis: findings from the North American Prodrome Longitudinal Study. Schizophr Bull. 2009;35:894-908.

16. Tsuang MT, Van Os J, Tandon R, et al. Attenuated psychosis syndrome in DSM-5. Schizophr Res. 2013;150:31-5. A summary that highlights the consensus of the DSM-5 Working Group on Schizophrenia Spectrum Disorders. Contains criteria and discussion of the issues.

17. McGlashan TH, Walsh BC, Woods SW. The psychosis-risk syndrome: Handbook for diagnosis and follow-up. New York: Oxford Univ Press; 2010.

18. Mohamed S, Rosenheck R, Swartz M, et al. Relationship of cognition and psychopathology to functional impairment in schizophrenia. Am J Psychiatry. 2008;165:978-87.

19. Nuechterlein KH, Subotnik KL, Ventura J, et al. The puzzle of schizophrenia: tracking the core role of cognitive deficits. Dev Psychopathol. 2012;24:529-36.

20. Saykin AJ, Shtasel DL, Gur RE, et al. Neuropsychological deficits in neuroleptic naive patients with first-episode schizophrenia. Arch Gen Psychiatry. 1994;51:124-31.

21. Aas M, Dazzan P, Mondelli V, et al. A systematic review of cognitive function in first-episode psychosis, including a discussion on childhood trauma, stress, and inflammation. Front Psychiatry. 2014;4:182.

22.• Bora E, Murray RM. Meta-analysis of cognitive deficits in ultra-high risk to psychosis and first-episode psychosis: Do the cognitive deficits progress over, or after, the onset of psychosis? Schizophr Bull. 2013. Addresses timely topics in the field related to the nature and onset of cognitive deficits in psychosis.

23. Seidman LJ, Giuliano AJ, Meyer EC, et al. Neuropsychology of the prodrome to psychosis in the NAPLS consortium: relationship to family history and conversion to psychosis. Arch Gen Psychiatry. 2010;67:578-88. Clinical high risk is associated with impaired neuropsychological functioning and poorer performance and family history increase risk of progression to psychosis.

24. Giuliano A, Li H, Mesholam-Gately R, et al. Neurocognition in the psychosis risk syndrome: a quantitative and qualitative review. Curr Pharm Des. 2012;18:399-415.

25. Fusar-Poli P, Deste G, Smieskova R, et al. Cognitive functioning in prodromal psychosis: a meta-analysis. Arch Gen Psychiatry. 2012;69:562-71.

26. Reichenberg A, Weiser M, Rapp MA, et al. Elaboration on premorbid intellectual performance in schizophrenia: premorbid intellectual decline and risk for schizophrenia. Arch Gen Psychiatry. 2005;62:1297-304.

27. Cannon M, Caspi A, Moffitt TE, et al. Evidence for earlychildhood, pan-developmental impairment specific to schizophreniform disorder: results from a longitudinal birth cohort. Arch Gen Psychiatry. 2002;59:449-56.

28. Fuller R, Nopoulos P, Arndt S, et al. Longitudinal assessment of premorbid cognitive functioning in patients with schizophrenia through examination of standardized scholastic test performance. Am J Psychiatry. 2002;159:1183-9.

29. Kirkpatrick B, Fenton WS, Carpenter WT, et al. The NIMHMATRICS consensus statement on negative symptoms. Schizophr Bull. 2006;32:214-9.

30. Irani F, Seligman S, Kamath V, et al. A meta-analysis of emotion perception and functional outcomes in schizophrenia. Schizophr Res. 2012;137:203-11.

31. Barbato M, Liu L, Penn DL, et al. Social cognition as a mediator between neurocognition and functional outcome in individuals at clinical high risk for psychosis. Schizophr Res. 2013;150:542-6.

32. Amminger GP, Schäfer MR, Klier CM, et al. Facial and vocal affect perception in people at ultra-high risk of psychosis, first-episode schizophrenia and healthy controls. Early Interv Psychiatry. 2012;6: 450-4.

33. Dickson H, Laurens KR, Cullen AE, et al. Meta-analyses of cognitive and motor function in youth aged 16 years and younger who subsequently develop schizophrenia. Psychol Med. 2012;42:74355 .

34. Kelleher I, Connor D, Clarke MC, et al. Prevalence of psychotic symptoms in childhood and adolescence: a systematic review and meta-analysis of population-based studies. Psychol Med. 2012;2012:1857-63. Provides an important developmental perspective reporting that psychotic symptoms are relatively common in young people, especially in childhood.

35. Kelleher I, Murtagh A, Clarke MC, et al. Neurocognitive performance of a community-based sample of young people at putative ultra high risk for psychosis: support for the processing speed hypothesis. Cogn Neuropsychiatry. 2013;18:9-25.

36. Reichenberg A, Caspi A, Harrington $\mathrm{H}$, et al. Static and dynamic cognitive deficits in childhood preceding adult schizophrenia: a 30year study. Am J Psychiatry. 2010;167:160-9.

37. Kelleher I, Murtagh A, Molloy C, et al. Identification and characterization of prodromal risk syndromes in young adolescents in the community: a population-based clinical interview study. Schizophr Bull. 2012;38:239-46.

38. Kaymaz N, Drukker M, Lieb R, et al. Do subthreshold psychotic experiences predict clinical outcomes in unselected non-helpseeking population-based samples? A systematic review and meta-analysis, enriched with new results. Psychol Med. 2012;42: 2239-53.

39. Gur RC, Calkins ME, Satterthwaite TD, et al. Neurocognitive growth charting in psychosis spectrum youths. JAMA Psychiatry. 2014.

40. Fusar-Poli P, McGuire P, Borgwardt S. Mapping prodromal psychosis: a critical review of neuroimaging studies. Eur Psychiatry. 2012;27:181-91.

41. Fusar-Poli P, Bonoldi I, Yung AR, et al. Predicting psychosis: a meta-analysis of transition outcomes in individuals at high clinical risk. Arch Gen Psychiatry. 2012;69:220-9.

42. Fusar-Poli P, Borgwardt S, Crescini A, et al. Neuroanatomical correlates of vulnerability to psychosis: a voxel-based metaanalysis. Neurosci Biobehav Rev. 2011;35:1175-85.

43. Carletti F, Woolley JB, Bhattacharyya S, et al. Alterations in white matter evident before the onset of psychosis. Schizophr Bull. 2012;38:1170-9.

44. Bloemen OJ, de Koning MB, Schmitz N, et al. White-matter markers for psychosis in a prospective ultra-high-risk cohort. Psychol Med. 2010;40:1297-304.

45. Borgwardt S, McGuire PK, Fusar-Poli P. Gray matters! Mapping the transition to psychosis. Schizophr Res. 2011;133: 63-7.

46. Smieskova R, Fusar-Poli P, Allen P, Bendfeldt K, et al. Neuroimaging predictors of transition to psychosis: a systematic 
review and meta-analysis. Neurosci Biobehav Rev. 2010;38:120722.

47. Crossley NA, Mechelli A, Fusar-Poli P, et al. Superior temporal lobe dysfunction and fronto-temporal dysconnectivity in subjects at risk of psychosis and in first-episode psychosis. Hum Brain Mapp. 2009;30:4129-37.

48. Fusar-Poli P, Meyer-Lindenberg A. Striatal presynaptic dopamine in schizophrenia, part II: meta-analysis of [18F]/[11C] DOPA PET studies. Schizophr Bull. 2013;39:33-42.

49. Howes O, Montgomery A, Asselin M, et al. Elevated striatal dopamine function linked to prodromal signs of schizophrenia. Arch Gen Psychiatry. 2009;66:13-20.

50. Howes OD, Bose SK, Turkheimer F, et al. Dopamine synthesis capacity before onset of psychosis: a prospective [18F]-DOPA PET imaging study. Am J Psychiatry. 2011;168:1311-7.

51. Tibbo P, Hanstock C, Valiakalayil A, Allen P. 3T proton MRS investigation of glutamate and glutamine in adolescents at high genetic risk for schizophrenia. Am J Psychiatry. 2004;61: 1116-8.
52. Fusar-Poli P, Howes OD, Allen P, et al. Abnormal prefrontal activation directly related to pre-synaptic striatal dopamine dysfunction in people at clinical high risk for psychosis. Mol Psychiatry. 2011;16:67-75.

53. Frommann I, Brinkmeyer J, Ruhrmann S, et al. Auditory P300 in individuals clinically at risk for psychosis. Int J Psychophysiol. 2008; 70:192-205.

54. Fusar-Poli P, Crossley N, Woolley J, et al. Gray matter alterations relted to P300 abnormalities in subjects at high risk for psychosis: longitudinal MRI-EEG study. Neuroimage. 2011;55:320-8.

55. Atkinson RJ, Michie PT, Schall U. Duration mismatch negativity and $\mathrm{P} 3 \mathrm{a}$ in first-episode psychosis and individuals at ultra-high risk of psychosis. Biol Psychiatry. 2012;71:98-104.

56. Shaikh M, Valmaggia L, Broome MR, et al. Reduced mismatch negativity predates the onset of psychosis. Schizophr Res. 2012;134:42-8.

57. Bodatsch M, Ruhrmann S, Wagner M, et al. Prediction of psychosis by mismatch negativity. Biol Psychiatry. 2011;69:959-66. 